[0212-7199 (2007) 24: 8; pp 365-368] ANALES DE MEDICINA INTERNA Copyright (C) 2007 ARAN EDICIONES, S.L.

AN. MED. INTERNA (Madrid) Vol. 24, N. ${ }^{\circ} 8$, pp. 365-368, 2007

\title{
Análisis de la actividad investigadora de los internistas gallegos a partir de los resultados de la oferta pública de empleo
}

\author{
R. RABUÑAL REY, R. MONTE SECADES, F. PAZ FUENTES, M. BAL ALVAREDO, \\ S. LÓPEZ LÓPEZ, C. ARIAS NÚÑEZ, J. GUERRERO LOMBARDÍA
}

Servicio de Medicina Interna. Complexo Hospitalario Xeral-Calde. Lugo

ANALYSIS OF THE INVESTIGATION ACTIVITY OF THE INTERNIST FROM THE PUBLIC BID OF EMPLOYMENT IN GALICIA, SPAIN

\begin{abstract}
RESUMEN
Objetivo: Estudiar la actividad investigadora de los internistas respecto a los otros especialistas médicos a partir de los datos de la Oferta Pública de Empleo.

Método: De la página web del Servicio Galego de Saude se han extraído los siguientes datos: especialidad, sexo, método de obtención del título de especialista, tesis doctoral y puntuación en lengua gallega, memoria profesional, experiencia profesional, docencia posgraduada y trabajos científicos.

Resultados: Se presentaron 355 médicos (33,2\% internistas, 66,8\% de otras especialidades médicas). No hubo diferencias entre ellos en cuanto a sexo, obtención del título por vía MIR, tesis doctoral, y puntuación en idioma gallego, experiencia profesional y docencia postgraduada. El número de publicaciones fue significativamente inferior en los internistas $(9,1 \pm 9,6 v s .15,7 \pm 12,1, \mathrm{p}<0,0001)$, tanto de forma global como cuando se analizó por grupos según los años de experiencia profesional. La obtención del título vía MIR se asoció a mayor número de publicaciones $(\mathrm{p}<0,001)$. La consecución de plaza se asoció a la puntuación de la memoria, la experiencia profesional y los trabajos publicados $(\mathrm{p}<$ $0,001)$. La realización de tesis doctoral se asoció a la obtención de plaza en hospitales universitarios $(\mathrm{p}<0,005)$.

Conclusión: Los internistas gallegos tienen menor actividad investigadora que los otros especialistas médicos. Es necesario, desde la medicina interna, promocionar la investigación y mejorar la formación de nuestros residentes en este aspecto.
\end{abstract}

\begin{abstract}
Objectives: The aim of the present study was to assess the investigative activity of the internists. Data were collected from the Public Bid of Employment (PBE) performed in Galicia in 2005, and compared with the rest of medical subspecialities.

Methods: The results from the PBE related to internal medicine and medical subspecialities are available in the web page of the Galician Service of Health. The following data were studied: speciality, sex, method of attainment of the specialist degree, thesis, and scores obtained in Galician language exam, professional experience, professional report, postgraduate teaching and published scientific papers.

Results: Data from 355 specialists were analyzed: 118 (33.2\%) were internists, and $237(66.8 \%)$ were medical subspecialists. There were no differences between internal medicine and the rest of subspecialities in terms of sex, method of attainment of the specialist degree, thesis, and scores obtained in Galician language exam, professional experience and postgraduate teaching. Internists presented fewer scientific papers than subspecialists, even when they were classified according to different levels of professional experience. Degrees obtained by the MIR system were associated with more publications $(p<0.001)$. The attainment of the job was associated to scores obtained in the professional report, professional experience, and published scientific papers $(p<0.001)$. The obtaining of the job was associated to the accomplishment of the doctoral thesis in the university teaching hospitals ( A Coruña, Santiago y Vigo, $p<0.005$ ).

Conclusions: The investigative activity of the internists in Galicia is inferior to the rest of the medical subspecialists. It seems necessary to stimulate the internists to developing scientific investigation and to improve those skills of the internal medicine residents.
\end{abstract}

KEY WORDS: Internal Medicine. Publications.

PALABRAS CLAVE: Medicina Interna. Publicaciones.

Rabuñal Rey R, Monte Secades R, Paz Fuentes F, Bal Alvaredo M, López López S, Arias Núñez C, Guerrero Lombardía J. Análisis de la actividad investigadora de los internistas gallegos a partir de los resultados de la oferta pública de empleo. An Med Interna (Madrid) 2007; 24: 365-368.

\section{INTRODUCCIÓN}

En los últimos años la Medicina Interna ha vivido un proceso de cambio, reflejado por un lado en la inquietud de los profesionales buscando nuevas vías de trabajo, como son las consultas de orientación de pacientes (1), las unidades de estancia corta (2) o la consultas quirúrgicas (3), y por otro desde la propia Sociedad Española de Medicina Interna (4), en el desarrollo de los grupos de trabajo y la elaboración del Estudio Socioprofesional (5) para caracterizar el trabajo realizado por los internistas dentro del Sistema Nacional de Salud (SNS). 
Un aspecto poco estudiado es la actividad investigadora de los internistas. A la recientemente celebrada oferta pública de empleo (OPE) se han presentado la gran mayoría de los internistas sin plaza fija en el SNS, aportando su experiencia profesional e investigadora para la baremación de méritos, lo cual nos permite hacer una evaluación de la misma mediante criterios objetivos. En el presente estudio nos proponemos estudiar la actividad investigadora de los internistas gallegos a partir de los datos presentados en la OPE (6), y compararla con la desarrollada por otros especialistas del área médica.

\section{MATERIAL Y MÉTODO}

En la pagina web del Servicio Galego de Saude (SERGAS) están publicados los listados de los resultados de la OPE extraordinaria correspondientes a Medicina Interna y resto de especialidades médicas. De ella se han extraído los datos referentes a los siguientes apartados: especialidad, sexo, método de obtención del título de especialista, tesis doctoral y puntuaciones alcanzadas en lengua gallega, memoria profesional, experiencia profesional, docencia posgraduada (ejercicio de funciones docentes sobre médicos residentes de la misma especialidad) y trabajos científicos. El baremo aplicado para la valoración de experiencia profesional y trabajos científicos se muestra en la tabla I. Dado que en el baremo se incluían diversas situaciones con diferente puntuación, para facilitar la interpretación de los resultados se dividió la calificación total obtenida en experiencia profesional por 3,6 (que corresponde a la puntuación que se obtendría por un año de trabajo en la misma categoría profesional en instituciones dependientes del SERGAS). Por la misma razón, en el apartado de trabajos científicos se dividió la puntuación obtenida por 0,25 (que corresponde al valor de una publicación en medios de difusión nacional). Los datos obte-

\begin{tabular}{|c|c|c|c|}
\hline \multicolumn{4}{|c|}{ TABLA I } \\
\hline \multicolumn{4}{|c|}{$\begin{array}{c}\text { BAREMO PARA LA VALORACIÓN DE EXPERIENCIA } \\
\text { PROFESIONAL Y TRABAJOS CIENTÍFICOS }\end{array}$} \\
\hline & & & Puntos \\
\hline \multirow{4}{*}{$\begin{array}{l}\text { Experiencia profesional } \\
\text { Máximo } 45 \text { puntos } \\
\text { (puntos por mes trabajad }\end{array}$} & $\begin{array}{l}\text { En instituciones sanitarias } \\
\text { dependientes del SERCAS }\end{array}$ & $\begin{array}{l}\text { En la misma } \\
\text { especialidad }\end{array}$ & 0,3 \\
\hline & & $\begin{array}{l}\text { En distinta } \\
\text { especialidad }\end{array}$ & 0,15 \\
\hline & En otros servicios de salud & $\begin{array}{l}\text { En la misma } \\
\text { especialidad }\end{array}$ & 0,1 \\
\hline & & $\begin{array}{l}\text { En distinta } \\
\text { especialidad }\end{array}$ & 0,05 \\
\hline \multirow{7}{*}{$\begin{array}{l}\text { Trabajos científicos } \\
\text { Máximo } 10 \text { puntos } \\
\text { (Sólo se valora a los tres } \\
\text { primeros firmantes) }\end{array}$} & Tesis doctoral & & 3 \\
\hline & Tesis doctoral cum laude & & 1 \\
\hline & Publicados en medios & Publicación en revista & 0,25 \\
\hline & de difusión nacional & Capitulo de libro & $\begin{array}{c}0,3 \\
1\end{array}$ \\
\hline & Publicados en medios de & Publicación en revista & 0,5 \\
\hline & difusión internacional & Capitulo de libro & 0,6 \\
\hline & & Libro completo & 2 \\
\hline
\end{tabular}

nidos se incluyeron en una tabla elaborada en Excel para su análisis estadístico mediante el programa SPSS 11.5.

\section{RESULTADOS}

Se analizaron los datos de 355 médicos; $118(33,2 \%)$ eran internistas, de los cuales consiguieron plaza $78(66,1 \%)$ y 237 $(66,8 \%)$ de otras especialidades médicas; la distribución detallada se muestra en la tabla II.

Inicialmente se compararon los datos correspondientes a medicina interna con el conjunto de los otros especialistas. No hubo diferencias en cuanto a sexo, obtención del título por vía MIR, realización de tesis doctoral, y puntuaciones en la prueba de idioma gallego, experiencia profesional e impartición de docencia postgraduada. Si hubo diferencia estadísticamente significativa a favor de las otras especialidades médicas en la puntuación de la memoria profesional y en el número de publicaciones (Tabla II, $\mathrm{p}<0,001$ y $\mathrm{p}<0,0001$, respectivamente); se comparó el número de publicaciones entre las diversas especialidades dos a dos comprobándose que la diferencia significativa respecto a medicina interna la establecían las especialidades de neurología y digestivo $(\mathrm{p}<0,001)$.

La obtención del título vía MIR se asoció a mayor número de publicaciones $(\mathrm{p}<0,001)$. Se realizó análisis por sexos no demostrándose diferencias en el método de obtención del titulo, realización de tesis doctoral ni puntuación en publicaciones; sí hubo diferencias en las puntuaciones obtenidas en experiencia profesional $(6,3 \pm 3,9$ en varones $v s .4,7 \pm 4,1$ en mujeres, $\mathrm{p}=0,002)$ y en docencia posgraduada $(2,5 \pm 1,8$ en varones vs. $1,6 \pm 1,8$ en mujeres, $\mathrm{p}=0,005)$. Asimismo se confirma una clara relación entre experiencia profesional y sexo masculino $(\mathrm{p}<0,005)$.

La tabla III muestra los datos ordenados en tres grupos, según la experiencia profesional en años, Se comprueba que por un lado el número de publicaciones aumenta significativamente con los años de experiencia $(\mathrm{p}=0,05$ al comparar el primer y segundo grupo y $\mathrm{p}=0,001$ al comparar el primer y tercer grupo). Para cada grupo los internistas obtienen en el número de publicaciones puntuaciones significativamente más bajas que el conjunto de los otros especialistas (menos de tres años $\mathrm{p}=0,001$, de 3 a 8 años $\mathrm{p}=0,006$ y más de 8 años $\mathrm{p}=0,02)$.

La consecución de plaza se asoció a la puntuación de la memoria, la experiencia profesional y los trabajos publicados $(\mathrm{p}<0,001)$. La realización de tesis doctoral se asoció a la obtención de plaza en los hospitales universitarios (Coruña, Santiago y Vigo, $\mathrm{p}<0,005)$. El examen de lengua gallega no discriminó a la hora de conseguir plaza.

\section{DISCUSIÓN}

El análisis realizado tiene dos limitaciones en relación con el método empleado. En primer lugar sólo incluye a los médicos que se presentaron a la OPE, por lo cual no están representados los que ya tenían plaza fija y los que trabajan en la medicina privada. En segundo lugar la actividad investigadora fue evaluada desde un punto de vista meramente cuantitativo, y no incluye toda la actividad científica ya que no se valoraron otras actividades como inclusión en grupos de trabajo, becas, publicaciones alternativas en la web, ni la calidad de las mis- 
TABLA II

RESULTADOS DE LA OPE EN MEDICINA INTERNA Y RESTO DE ESPECIALIDADES MÉDICAS

\begin{tabular}{|c|c|c|c|c|c|c|c|c|c|}
\hline & Número & $\begin{array}{c}\text { Sexo } \\
\text { (\% varón) }\end{array}$ & $\begin{array}{c}\text { Título } \\
\text { (\% vía MIR) }\end{array}$ & $\begin{array}{l}\text { Tesis } \\
(\%)\end{array}$ & Memoria* & Idioma* & $\begin{array}{l}\text { Experiencia } \\
(\text { (años)* }\end{array}$ & $N^{0}$ publicaciones* & Docencia* \\
\hline Medicina Interna & 118 & 55,9 & 86,4 & 15,2 & $81,6 \pm 8,6$ & $3,7 \pm 0,8$ & $5,8 \pm 4,4$ & $9,1 \pm 9,6$ & $1,9 \pm 2$ \\
\hline $\begin{array}{l}\text { Otras especialidades } \\
\text { Cardiología } \\
\text { Digestivo } \\
\text { Endocrinología } \\
\text { Geriatría } \\
\text { Neumología } \\
\text { Neurología } \\
\text { Oncología } \\
\text { Reumatología }\end{array}$ & $\begin{array}{c}237 \\
50 \\
36 \\
26 \\
11 \\
30 \\
36 \\
25 \\
23\end{array}$ & $\begin{array}{c}63,3 \\
78 \\
69,4 \\
42,3 \\
54,5 \\
66,7 \\
52,8 \\
60 \\
65,2\end{array}$ & $\begin{array}{c}88,6 \\
74 \\
69,4 \\
92,3 \\
63,6 \\
96,7 \\
100 \\
96 \\
91,3\end{array}$ & $\begin{array}{c}22,8 \\
20 \\
19,4 \\
34,6 \\
9,1 \\
10 \\
38,9 \\
12 \\
30,4\end{array}$ & $\begin{array}{c}86,7 \pm 7,4 \\
81,1 \pm 10,7 \\
88,9 \pm 5,3 \\
90,3 \pm 2,4 \\
89,4 \pm 2,7 \\
87,5 \pm 5,7 \\
90,5 \pm 0,9 \\
82,5 \pm 4,7 \\
87,2 \pm 8,4\end{array}$ & $\begin{array}{c}3,8 \pm 0,7 \\
3,7 \pm 0,7 \\
3,9 \pm 0,7 \\
4 \pm 0,7 \\
3,8 \pm 0,6 \\
3,8 \pm 0,6 \\
3,8 \pm 0,6 \\
3,6 \pm 0,9 \\
3,9 \pm 0,9\end{array}$ & $\begin{array}{c}5,6 \pm 3,9 \\
5,9 \pm 3,6 \\
5,1 \pm 4 \\
6,2 \pm 4 \\
3,8 \pm 4,3 \\
5,3 \pm 3,4 \\
5,3 \pm 4,1 \\
5,1 \pm 3,1 \\
7,4 \pm 4,5\end{array}$ & $\begin{array}{c}15,7 \pm 12,1 \\
14,6 \pm 13,2 \\
21,7 \pm 11,9 \\
9,8 \pm 8 \\
9,9 \pm 12 \\
14,2 \pm 11,8 \\
25,6 \pm 9,9 \\
7 \pm 4,8 \\
13,7 \pm 9,3\end{array}$ & $\begin{array}{c}2,3 \pm 1,8 \\
2,3 \pm 1,5 \\
2 \pm 1,9 \\
1,2 \pm 1,9 \\
3,2 \pm 1,7 \\
2,4 \pm 1,6 \\
2,2 \pm 1,8 \\
2,4 \pm 1,6 \\
3,1 \pm 1,9\end{array}$ \\
\hline Total & 355 & 60,8 & 87,9 & 20,3 & $85 \pm 8,2$ & $3,8 \pm 0,7$ & $5,7 \pm 4$ & $13,5 \pm 11,8$ & $2,1 \pm 1,9$ \\
\hline
\end{tabular}

* Media \pm desviación estándar.

TABLA III

COMPARACIÓN DEL NÚMERO DE PUBLICACIONES ENTRE MEDICINA INTERNA Y EL CONJUNTO DE OTRAS ESPECIALIDADES SEGÚN LA EXPERIENCIA PROFESIONAL EN AÑOS

\begin{tabular}{|c|c|c|c|c|c|c|c|c|c|c|}
\hline & \multicolumn{2}{|c|}{ Número } & \multicolumn{2}{|c|}{ Sexo (\% varón) } & \multicolumn{2}{|c|}{ Título vía MIR(\%) } & \multicolumn{2}{|c|}{ Tesis (\%) } & \multicolumn{2}{|c|}{ Publicaciones $\left(N^{0}\right)^{*}$} \\
\hline & $\mathrm{Ml}$ & Otras & Ml & Otras & $\mathrm{Ml}$ & Otras & $\mathrm{Ml}$ & Otras & Ml & Otras \\
\hline $\begin{array}{l}\text { Hasta } 3 \text { años } \\
\text { Entre } 3 \text { y } 8 \text { años } \\
\text { Mas de } 8 \text { años } \\
\text { Total }\end{array}$ & $\begin{array}{c}44 \\
32 \\
42 \\
118\end{array}$ & $\begin{array}{c}74 \\
91 \\
72 \\
237\end{array}$ & $\begin{array}{c}38,6 \\
62,5 \\
69 \\
55.9\end{array}$ & $\begin{array}{l}52,1 \\
65,9 \\
70,8 \\
63,3\end{array}$ & $\begin{array}{l}77,3 \\
100 \\
88,1 \\
86,4\end{array}$ & $\begin{array}{c}90,5 \\
97,8 \\
75 \\
88,6\end{array}$ & $\begin{array}{l}2,3 \\
18,7 \\
26,2 \\
15,2\end{array}$ & $\begin{array}{l}10,8 \\
18,7 \\
40,3 \\
22,8\end{array}$ & $\begin{array}{c}5,9 \pm 7,4 \\
10,5 \pm 9,9 \\
11,3 \pm 10,6 \\
9,1 \pm 9,6\end{array}$ & $\begin{array}{l}12,7 \pm 11,6 \\
17,6 \pm 13,2 \\
16,2 \pm 10,7 \\
15,7 \pm 12,1\end{array}$ \\
\hline
\end{tabular}

* Media \pm desviación estándar.

mas. Asimismo, en el apartado de experiencia profesional sólo se incluyó el trabajo dentro del SNS, no otro tipo de experiencia.

A pesar de todos estos posibles sesgos, creemos que los datos son representativos de la situación de los internistas gallegos incorporados en los últimos 20 años, dado el elevado número de participantes (según el Estudio Socioprofesional (5) hay en Galicia 212 internistas, y se presentaron a la OPE 118 , el 55,7\% del total), y la aplicación del mismo baremo de méritos para todos.

Hasta ahora se habían realizado dos tipos de aproximaciones al análisis de la producción científica en medicina en España. Por un lado estudios bibliométricos (7-9), útiles para valorar tendencias generales, sobre todo comparativas entre áreas temáticas o países, que sin embargo presentan las limitaciones de evaluar únicamente las publicaciones incluidas en los correspondientes índices analizados, y no identificar la especialidad de los autores. Esto es especialmente relevante en el área de la Medicina Interna, dado que muchos especialistas publican en revistas de la especialidad correspondiente al área de trabajo que desarrollan, no en revistas generales de Medicina Interna. Otra aproximación es la realizada a través de la participación en congresos de la especialidad $(10,11)$, lo cual permite un análisis más dirigido de la actividad dentro de la propia especialidad, pero no aclara si dicha actividad investigadora ha culminado en su publicación en una revista tras la necesaria "revisión por pares". Ambas aproximaciones son generales y no evalúan la actividad de cada facultativo por separado, tal y como se hace en nuestro trabajo. Por otra parte no valoran libros, ni publicaciones no indexadas, que sí se han incluido en el baremo de la OPE. Es probable, por ultimo, que la aportación de meritos en la OPE, por ser voluntaria e interesada, haya sido más exhaustiva que la obtenida por otros medios.

Es significativa la confirmación de que los internistas publican menos que el resto de los especialistas, tanto de forma global como para cada grupo de edad. Esto probablemente es debido a la suma de múltiples factores: una actividad predominantemente clínica, cercana a la cabecera del enfermo pero alejada del laboratorio, una mayor presencia de los internistas en hospitales comarcales respecto a los de tercer nivel, o en áreas clínicas de gran presión asistencial, como son los Servicios de Urgencias, y posiblemente también una menor "tradición" publicadora, a lo que se añaden problemas estructurales (equipamiento) y de financiación. Seguramente influye también la menor cantidad de revistas específicamente dedicadas a la medicina interna respecto a otras especialidades, lo que supone una dificultad añadida para publicar. Como ejemplo en España hay 14 revistas de medicina interna, mientras que existen 16 en neurología (12), para un número de especialistas mucho menor. De todas formas la actividad investigadora es superior a la de otros colectivos de nuestra 
comunidad autónoma; sólo el 19,5\% de los internistas no aportaron ninguna publicación, frente al $62,8 \%$ de los médicos de Urgencias Hospitalarias (13) o el 82,4\% de los médicos de Atención Primaria (14); probablemente en estos colectivos el bajo nivel de publicación tenga las mismas causas que las expuestas para los internistas, pero llevadas al extremo.

Se ha sugerido que las mujeres presentan niveles de producción científica más bajos (15), achacable a una posible discriminación de género en el trabajo y/o mayor dedicación a tareas domésticas y familiares. En nuestro caso, aunque la proporción de mujeres es superior entre los internistas que en las otras especialidades, no se han demostrado diferencias en la cantidad de publicaciones que justifiquen esta aseveración. Se han comunicado resultados similares en otros colectivos médicos $(13,14)$.

Un dato llamativo es el que un $22,7 \%$ de los internistas con menos de 3 años de experiencia no acreditaran la obtención del título por la vía MIR, frente al 9,5\% en el caso de los otros especialistas $(p=0,05)$; esto podría justificar parte de la diferencia en número de publicaciones, dado que la obtención del título por la vía MIR se asoció a mayor número de publicaciones. Una posible explicación sería que en este grupo se incluyeran por un lado médicos con títulos obtenidos en el extranjero convalidados y por otro que los que ya obtuvieron

\section{Bibliografía}

1. Sanchís-Bayarri Bernal V, Rull Segura S, Moral López L, García Ferrer E, Aparisi Romero J, Escandón Álvarez J. Consulta de orientación de pacientes: una iniciativa para reducir listas de espera. Rev Clin Esp 2003; 203: 133-5.

2. De la Iglesia Martínez F, Pellicer Vázquez C, Ramos Polledo V, Castro Romero B, Rodríguez Sotillo A, Diz-Lois Martínez F. La unidad de corta estancia médica (UCEM) de la Coruña: nuestra experiencia. An Med Interna (Madrid) 1997; 14: 125-7.

3. Monte Secades R, Rabuñal Rey R, Rigueiro Veloso MT, García Pais MJ, Casariego Vales E, Guerrero Lombardía J. Papel del internista como consultor de servicios quirúrgicos. Rev Clin Esp 2004; 204: 34550 .

4. González de la Puente MA, Pujol R, Conté P. La Sociedad Española de Medicina Interna (SEMI). Logros alcanzados y retos pendientes. Septiembre 2006. Rev Clin Esp 2006; 206: 471-3.

5. Estudio Socioprofesional de la Medicina Interna en España: prospectiva 2010. Madrid: Sociedad Española de Medicina Interna (SEMI), 2005.

6. Listaxe definitiva de superación da fase de selección do proceso extraordinario de consolidación de emprego, consultado el 3/12/2006 en https://www.sergas.es/gal/personal/ConsolidacionEmpleo.asp?Tipo=1 \&Color=

7. Gómez Caridad I, Fernández Muñoz MT, Bordons Gangas M, Morillo Ariza F. La producción científica española en Medicina en los años 1994-1999. Rev Clin Esp 2004; 204: 75-88.

8. Camí J, Suñén Piñol E, Méndez Vásquez R. Mapa bibliométrico de España 1994-2002: biomedicina y ciencias de la salud. Med Clin (Barc) 2005; 124: 93-101.

9. San Román Terán C, Guil García M, Medina Delgado P, Montero Rivas L, Ramos Cantos C, Martín Pérez M, et al. ¿Qué comunicamos los internistas? Estudio bibliométrico de los Servicios de Medicina una baja puntuación en la fase de oposición, desistieran posteriormente de aportar nuevos méritos.

El escaso número de tesis doctorales realizadas (sólo el $15,2 \%$ de los internistas acreditaron la realización de una tesis) demuestra la falta de una adecuada conexión entre los internistas y la Universidad. Este dato es corroborado en un libro recientemente editado por la SEMI (16), en donde sólo se recogen 8 tesis presentadas por internistas en las Universidades gallegas, aspecto en el que habría que incidir en las instancias oportunas para mejorar esta situación

En conclusión, con los datos extraídos de la OPE podemos asegurar que la actividad investigadora de los internistas gallegos es manifiestamente mejorable. Es importante invertir esta tendencia, ya no sólo por motivos de prestigio social o profesional, sino también por que el objetivo primario de toda investigación biomédica es "favorecer el interés del enfermo para proporcionarle una vida más larga y mejor" (17). Debemos, desde la medicina interna, estimular actividades de promoción de la investigación y mejorar la formación de nuestros residentes en este aspecto. Sería deseable la acreditación de un mínimo de producción científica de calidad en los hospitales docentes, así como la creación de redes colaborativas entre los diversos servicios de medicina interna y de estos con las universidades.
Interna de España. 2. Publicaciones. XXVII Congreso Nacional de la Sociedad Española de Medicina Interna, Salamanca, 25-28 de octubre de 2006. Rev Clin Esp 2006; 206 (Supl. 3): 137.

10. Montes Santiago J, Pérez Álvarez R. Aportación científica de la medicina interna hospitalaria gallega a congresos nacionales e internacionales de su especialidad. An Med Interna (Madrid) 2002; 19: 567-70.

11. San Román Terán C, Montero Rivas L, Ramos Cantos C, Martín Pérez M, Pérez Díaz J, Medina Delgado P, et al. ¿Qué comunicamos los internistas?. Estudio bibliométrico de los Servicios de Medicina Interna de España. 1. Comunicaciones y tesis doctorales. XXVII Congreso Nacional de la Sociedad Española de Medicina Interna, Salamanca, 25-28 de octubre de 2006. Rev Clin Esp 2006; 206 (Supl. 3): 137-8.

12. González de Dios J, Sempere AP, Aleixandre-Benavent R. Las publicaciones biomédicas en España a debate (I): estado de las revistas neurológicas. Rev Neurol 2007; 44: 32-42.

13. Bugarín González R, Galego Feal P, Gutiérrez Molina A. La actividad investigadora de los profesionales de urgencias en la Comunidad Autónoma de Galicia. Emergencias 2006; 18: 110-5.

14. Bugarín González R, Queiro Verdes T, Santiago Pérez I, Gutiérrez Molina A, García Quintans A, Galego Feal P. Perfil del medico de familia que investiga: extrapolación a partir de la oferta publica de empleo de Galicia. Atención Primaria, 2005; 36: 526-7.

15. Rusell JM. Los indicadores de producción científica por genero - un caso especial. Tercer taller de obtención de indicadores bibliométricos. Madrid, 3-5 de marzo de 2003. Consultado el 5/2/2007 en http://www.ricyt.edu.ar/interior/normalizacion/III_bib/Rusell.pdf

16. 1976-2004. La investigación española en Medicina Interna a través de las Tesis Doctorales. Madrid: Sociedad Española de Medicina Interna (SEMI), 2006.

17. Rozman C. Reflexiones sobre la investigación biomédica en España. Med Clin (Barc) 2003; 120: 19-23. 